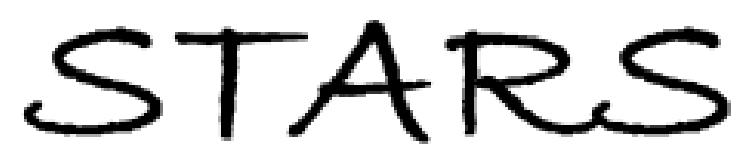

University of Central Florida

STARS

$1-1-2000$

\title{
Interface structures in GaAs wafer bonding: Application to compliant substrates
}

\author{
R. R. Vanfleet \\ M. Shverdin \\ J. Silcox \\ Z. H. Zhu \\ Y. H. LO
}

Find similar works at: https://stars.library.ucf.edu/facultybib2000 University of Central Florida Libraries http://library.ucf.edu

This Article is brought to you for free and open access by the Faculty Bibliography at STARS. It has been accepted for inclusion in Faculty Bibliography 2000s by an authorized administrator of STARS. For more information, please contact STARS@ucf.edu.

\section{Recommended Citation}

Vanfleet, R. R.; Shverdin, M.; Silcox, J.; Zhu, Z. H.; and Lo, Y. H., "Interface structures in GaAs wafer bonding: Application to compliant substrates" (2000). Faculty Bibliography 2000s. 2843.

https://stars.library.ucf.edu/facultybib2000/2843

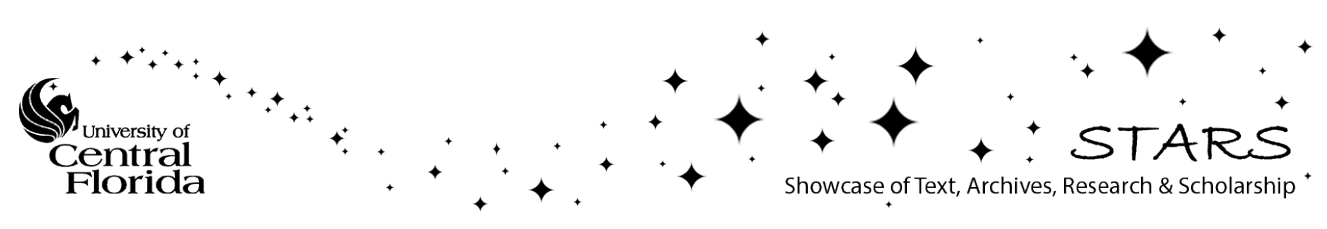




\section{Interface structures in GaAs wafer bonding: Application to compliant substrates}

Cite as: Appl. Phys. Lett. 76, 2674 (2000); https://doi.org/10.1063/1.126440

Submitted: 05 January 2000 . Accepted: 13 March 2000 . Published Online: 01 May 2000

R. R. Vanfleet, M. Shverdin, J. Silcox, Z. H. Zhu, and Y. H. Lo

\section{Applied Physics Letters}

Mid-IR and THz frequency combs special collection 


\title{
Interface structures in GaAs wafer bonding: Application to compliant substrates
}

\author{
R. R. Vanfleet, ${ }^{\text {a) }}$ M. Shverdin, ${ }^{\text {b) }}$ and J. Silcox \\ School of Applied and Engineering Physics, Cornell University, Ithaca, New York 14853 \\ Z. H. Zhu and Y. H. Lo \\ Department of Electrical Engineering, Cornell University, Ithaca, New York 14853
}

(Received 5 January 2000; accepted for publication 13 March 2000)

\begin{abstract}
The interface of direct bonded GaAs to GaAs has been studied by scanning transmission electron microscopy and electron energy loss spectroscopy. Voids are seen along the boundary with most being partially filled with a gallium particle. Two general sizes of voids are seen. The large voids $(d \sim 45 \mathrm{~nm})$ are distributed in an approximately linear relationship and the smaller $(d \sim 12 \mathrm{~nm})$ randomly. In compliant substrates, one of the layers is made thin $(\leqslant 10 \mathrm{~nm})$ and twisted $\sim 45^{\circ}$. The larger voids often extend past this thin compliant layer, but no evidence of granularity of the epitaxial film is observed. (c) 2000 American Institute of Physics. [S0003-6951(00)00119-4]
\end{abstract}

Direct wafer bonding is a potential avenue for significant advances in device applications either through direct bonding of differing materials ${ }^{1-4}$ or production of compliant substrates $^{5,6}$ for growth of lattice mismatched materials. Interface defects [seen as white spots in bright field transmission electron microscopy (TEM) images] are a common observation when GaAs is used as one of the bonding materials ${ }^{1,2,4,7}$ but have not yet been identified in GaAs to GaAs bonding. Voids would be expected if bubbles, due to surface particulates or varying surface morphologies, are trapped during interface formation. Gallium precipitates or gallium enrichment at the interface might be expected if arsenic leaks out (due to higher volatility) during the bonding process.

Some of the observed defects are large and in the case of compliant substrate production penetrate through the thin compliant layer. Kopperschmidt et al. ${ }^{7}$ suggested these large defects act as pinholes, giving rise to multigranular films through the nucleation of subsequent epitaxial growth on the underlying bulk layer. In this letter, we report evidence from scanning transmission electron microscopy (STEM) techniques that the defects are combined void/Ga precipitate structures reflecting the departure of As from the interface during the interface formation process. On the other hand, we have no evidence for multigranularity, which may reflect differences in the preparation procedures between our approach and that given by Kopperschmidt et al. ${ }^{7}$

A $10 \mathrm{~nm}$ GaAs compliant layer was made and bonded to a handling wafer according to the description given in Ref. 6. A $500 \mathrm{~nm}$ thickness of lattice matched $\mathrm{InGaP}$ was then grown at $640^{\circ} \mathrm{C}$ on top of the $10 \mathrm{~nm}$ GaAs layer. Because the growth layers are lattice matched to the GaAs, no effects of lattice mismatch were expected or seen. Cross-sectional specimens were made by mechanical polishing using the

${ }^{a}$ Present address: Advanced Materials Processing and Analysis Center, University of Central Florida, Orlando, Florida 32826; electronic mail: vanfleet@physics.ucf.edu

${ }^{b}$ Present address: Edward L. Ginzton Laboratory, Stanford University, Stanford, California 94305. wedge technique ${ }^{8}$ followed up by argon ion milling. Plan view specimens were prepared by etching off the InGaP growth layer, mounting on a copper washer, mechanically polishing the bulk wafer side to $<20 \mu \mathrm{m}$, and then etching with a citric acid solution ${ }^{9}$ from the bulk wafer side until perforated.

STEM images are formed by the display of various detector signals resulting from scanning a focused electron beam $(\sim 0.2 \mathrm{~nm}$ in diameter for the Cornell UHV STEM) over an electron transparent specimen. The annular dark field (ADF) mode collects those electrons that have been scattered to large angles (using an area detector with a hole in the center) and is similar to Rutherford scattering, i.e., is dependent on the atomic numbers $(\mathrm{Z})$ of the atoms as well as the number of atoms. Thus, it is commonly referred to qualitatively as Z-contrast imaging. There are however, effects due to strain, roughness, sample thickness, crystal orientation, and other disorder in the specimen that can often preclude simple quantitative interpretation of the observed intensities. ${ }^{10-12}$

Because the electron beam is focused to a small probe, electron energy loss spectroscopy (EELS) data can be collected with high spatial resolution, by means of a magnetic prism. ${ }^{13}$ This allows compositional information to be determined, based on element specific core energy losses, at the subnanometer scale, as well as maps of the specimen thickness, based on plasmon scattering. The strongest feature of a good EELS spectrum is the zero-loss peak (electrons that have lost no energy) followed by the plasmon peak (due to collective excitations of the valence electrons). The core edges are significantly weaker than the zero-loss or plasmon features and sit on a background composed of the tails of the plasmons and other core edges. The number of counts above the background level in a core edge spectrum is proportional to the number of atoms of that element at the probe position. The ratio of the first plasmon intensity to the zero loss intensity gives the thickness of the material in units of mean free path for plasmon scattering. Images can be obtained using only electrons that have lost a plasmon energy or that have lost no energy. The ratio of these images forms a thickness 

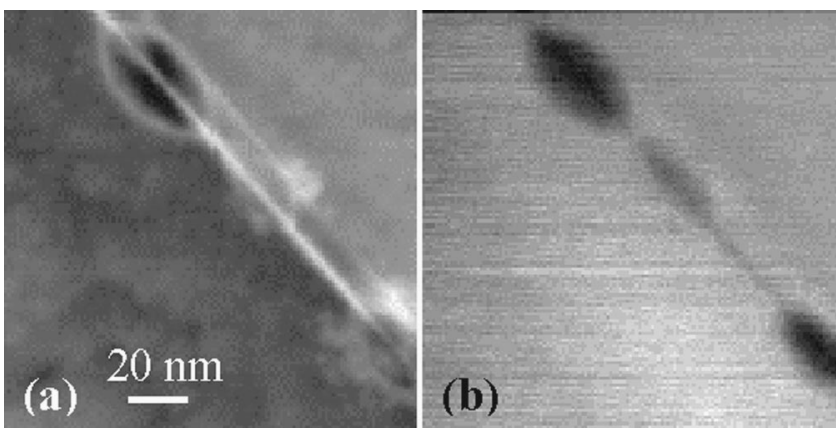

FIG. 1. (a) is a cross-sectional annular dark field image of the compliant layer. The lower left is the bulk GaAs wafer and the upper right is the InGaP. The bright line is the twist boundary upon which three defects are seen. (b) is a thickness map of the region shown in (a) and clearly shows the interface defects to be voids.

map. Such maps are often of value even in samples of uneven composition.

Figure 1(a) shows an annular dark field (ADF) image of a cross-sectional view of the bonded layer. The brighter area at the top right is the InGaP growth layer. Its brightness reflects the high $\mathrm{Z}$ indium content. The line $\sim 10 \mathrm{~nm}$ below the $\mathrm{InGaP}$ is the bonded interface which is also brighter. This is probably due to the disorder that accommodates the relative twist of the two GaAs layers. A series of defects are seen along the interface. In general the interface defects are of lower intensity indicating a region of lower atomic number or a void. Some of the defects show inclusions or portions filled with material that scatters similarly to the GaAs. The plan view images show these inclusions to be a common feature. Figure 1(b) shows a thickness map of the region shown in Fig. 1(a). The interface defects show up as decreases in the projected thickness of the material, again indicative of a void.

Figure 2 shows an ADF image of the plan view specimen. The bright spots result from inadequate rinsing after etching with citric acid. The black spots are the interface defects with the lower intensity indicating void structures. At

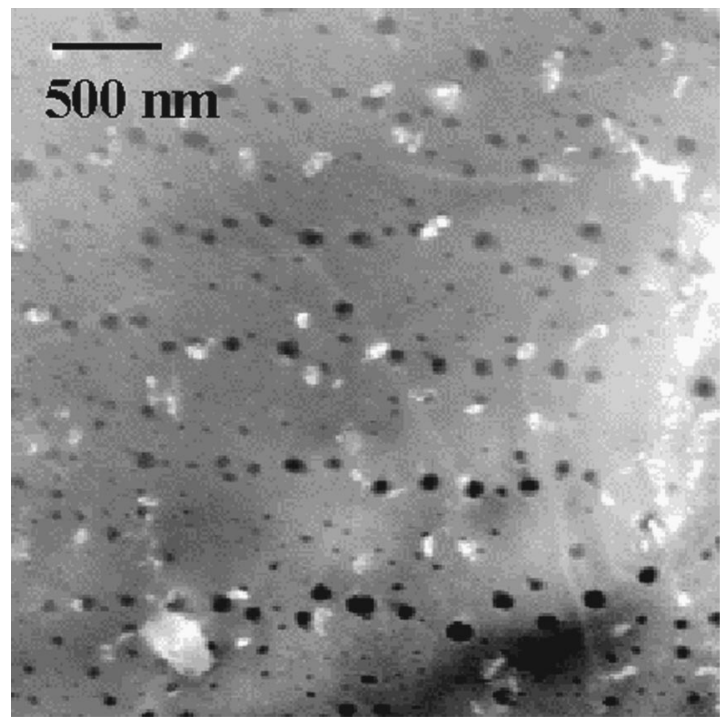

FIG. 2. ADF plan view showing interface defects. Large defects (black spots) are arranged in an approximately linear manner and the small defects randomly positioned. The bright spots are residual citric acid from the etching process.

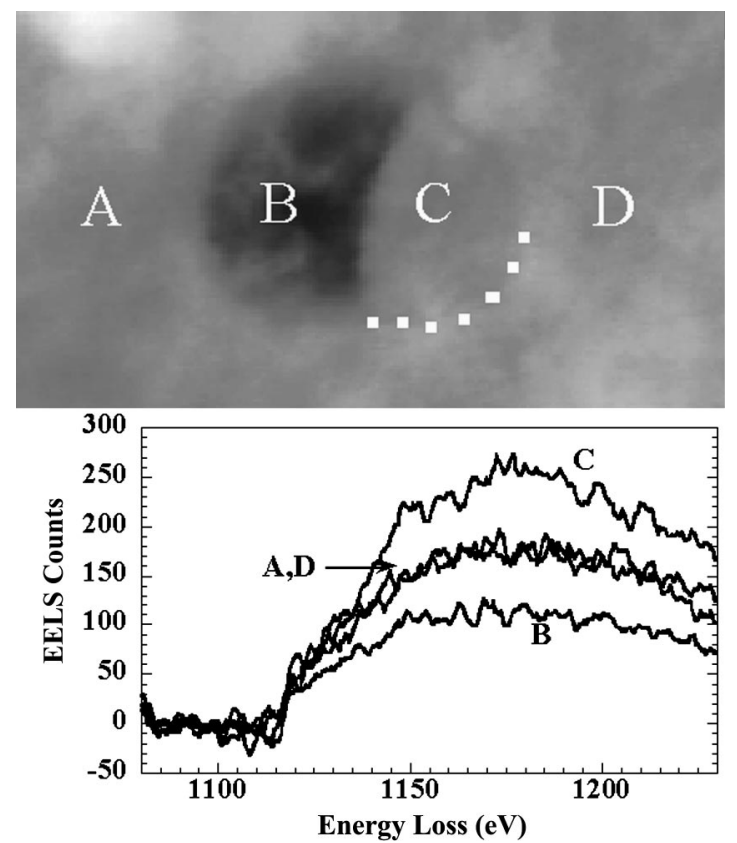

FIG. 3. The stripped EELS gallium L edge is shown at the four locations indicated in the top figure. The counts under the edge are proportional to the gallium content. Region B shows a decrease in gallium and C an increase.

this magnification, two general sizes of interface defects are apparent. The larger defects are $\sim 45 \mathrm{~nm}$ in diameter and appear to have formed along lines. These lines vary from 10 to $20^{\circ}$ off of the 110 directions of the handling crystal depending upon location on the specimen. A second smaller defect, $d \sim 12 \mathrm{~nm}$, is also seen with positions that appear to be random. The size of the defects in the direction normal to the wafer is slightly smaller than half the diameter. Thus, most of the larger diameter defects extend through the $10 \mathrm{~nm}$ compliant layer. The linear positioning of these larger defects is not yet understood.

Figure 3 shows a higher magnification plan view ADF image of a typical void structure. Most voids are irregular in shape, being partially filled by inclusions which scatter similarly to the GaAs. A portion of the edge of this inclusion is highlighted with spots in Fig. 3. Several of the inclusions show moiré fringes indicating the inclusions are crystalline. Figure 3 shows the gallium L edge EELS spectra (after background subtraction) at four points indicated in the image. The counts are proportional to the gallium content and show an increase in gallium content at the inclusion (point C). Additionally, EELS scans do not show any significant variation of other materials across the specimen. This data leads to the general conclusion that the inclusions are elemental gallium, although there were only a small number of inclusions where EELS was used directly to confirm this conclusion. Arsenic, which has a higher vapor pressure than gallium is presumably lost during the bonding process, leaving excess gallium along the bonded interface. The lower gallium content at point B is consistent with the void interpretation arising from the lower ADF intensity.

A recent letter ${ }^{7}$ suggested that the low number of defects observed in specimens similar to the one studied here, ${ }^{3,5,6}$ arose from a multigranular nature of the film. They ${ }^{7}$ observed a multigranular film on their thin compliant layer with grains growing either epitaxially on the substrate or on the 
twisted layer. We observed no granularity in the epitaxial layer. We have however seen evidence that the twisted layer is very delicate and even careful handling when the compliant layer is exposed can be enough to strip the thin layer off the substrate. Thus, an alternate possibility for the multigranular observations is that the ultrasonically enhanced etching used to remove down to the compliant layer also damaged the layer, leaving holes in the compliant layer sufficient to allow nucleation on the substrate resulting in a multigranular film.

The reason for the linear arrangement of the larger defects is not clear at this point, but some speculation can be put forth. Potentially, the large defects could indicate the escape path for the arsenic during the bonding process. These large defects may be forming at step edges. X-ray scattering measurements showed that the two wafers had a relative tilt from the surface normal of $0.6^{\circ}$. This tilt and the line spacing of $\sim 500 \mathrm{~nm}$ would imply steps of $5 \mathrm{~nm}$, which seem too large to be likely. Scratches in the wafer surface are perhaps a more likely explanation. Scratches, even very small ones, would provide an escape channel for the arsenic vapor as well as a lack of material, which would result in voids larger than would be justified by the loss of arsenic.

In summary, ADF STEM images of interface defects in high angle twist bonded GaAs supported by some EELS observations suggest these are voids with crystalline gallium precipitates at the edges. Most of the interface defects are small ( $\sim 12 \mathrm{~nm}$ in diameter by $\sim 5 \mathrm{~nm}$ in height), but a small number are seen to be larger $(\sim 45 \mathrm{~nm}$ in diameter) and often extend through the thin $(10 \mathrm{~nm})$ compliant layer. These larger defects are arranged in an approximately linear fashion; the exact reason for this relationship is not clear. Despite the large defects or pinholes through the compliant layer the epitaxial layer is single crystal.

Support from Earl Kirkland and Mick Thomas is gratefully acknowledged. This research was supported by U.S. Air Force Grant No. F49620-95-1-0427. Z. H. Zhu and Y. H. Lo acknowledge the research support from Office of Naval Research, National Science Foundation, and DARPA. The Cornell STEM was acquired through the NSF (Grant No. DMR-8314255) and is operated by the Cornell Center for Materials Research (NSF Grant No. DMR-9632275).

${ }^{1}$ P. Kopperschmidt, G. Kastner, St. Senz, D. Hesse, and U. Gosele, Appl. Phys. A: Solids Surf. 64, 533 (1997).

${ }^{2}$ G. Patriarche, F. Jeannes, J.-L. Oudar, and F. Glas, J. Appl. Phys. 82, 4892 (1997)

${ }^{3}$ Z. H. Zhu, R. Zhou, F. E. Ejeckam, Z. Zhang, J. Zhang, J. Greenberg, Y. H. Lo, H. Q. Hou, and B. E. Hammons, Appl. Phys. Lett. 72, 2598 (1998).

${ }^{4}$ P. Kopperschmidt, S. Senz, G. Kastner, D. Hesse, and U. Gosele, Appl. Phys. Lett. 72, 3181 (1998).

${ }^{5}$ F. E. Ejeckam, Y. H. Lo, and S. Subramanian, Appl. Phys. Lett. 70, 1685 (1997).

${ }^{6}$ F. E. Ejeckam, M. L. Seaford, Y. H. Lo, H. Q. Hou, and B. E. Hammons, Appl. Phys. Lett. 71, 776 (1997).

${ }^{7}$ P. Kopperschmidt, St. Senz, R. Scholz, and U. Gosele, Appl. Phys. Lett. 74, 374 (1999).

${ }^{8}$ S. J. Klepeis, J. P. Benedict, and R. M. Anderson, Specimen Preparation for Transmission Electron Microscopy (Materials Research Society, Pittsburgh, PA, 1988), p. 179

${ }^{9}$ S. Hillyard, Ph.D. Thesis, Cornell University, 1996.

${ }^{10}$ A. V. Crewe, J. P. Langmore, and M. S. Isaacson, Physical Aspects of Electron Microscopy and Microbeam Analysis, edited by B. M. Siegel and D. K. Beaman (Wiley, New York, 1975), p. 47.

${ }^{11}$ S. J. Pennycook and D. E. Jesson, Ultramicroscopy 37, 14 (1991).

${ }^{12}$ R. F. Loane, P. Xu, and J. Silcox, Ultramicroscopy 40, 121 (1992).

${ }^{13}$ R. F. Egerton, Electron Energy-Loss Spectroscopy in the Electron Microscope (Plenum, New York, 1986). 